\title{
Ataxic Gait
}

National Cancer Institute

\section{Source}

National Cancer Institute. Ataxic Gait. NCI Thesaurus. Code C3834.

Ambulatory abnormalities caused by an impairment of voluntary muscle coordination, most often and significantly affecting gait. 